\title{
Nutrient deprivation induces apoptosis of nucleus pulposus cells via activation of the BNIP3/AIF signalling pathway
}

\author{
JIE LIU ${ }^{1}$, CHAO YUAN ${ }^{2}$, LUQIAO PU ${ }^{2}$ and JIAN WANG ${ }^{2}$ \\ ${ }^{1}$ Department of Orthopaedics, The First People's Hospital of Yunnan, Kunming, Yunnan 650032; ${ }^{2}$ Department of Orthopaedics, \\ Xinqiao Hospital, The Third Military Medical University, Chongqing 400038, P.R. China
}

Received December 1, 2016; Accepted June 29, 2017

DOI: $10.3892 / \mathrm{mmr} .2017 .7550$

\begin{abstract}
Nutrient deprivation (ND)-induced nucleus pulposus (NP) cell death serves an important role in intervertebral disc degeneration disease. However, the underlying mechanisms have yet to be thoroughly elucidated. The present study created a cell culture model under ND conditions to investigate the roles of the nutrient-sensitive protein B-cell lymphoma 2/adenovirus E1B $19 \mathrm{kDa}$-interacting protein (BNIP3) and the mitochondrial pro-death protein apoptosis-inducing factor (AIF) in the death pathway of NP cells. The present study demonstrated that cells subjected to ND for up to $72 \mathrm{~h}$ exhibited a time-dependent increase in cell death and decrease in mitochondrial membrane potential $(\Delta \psi \mathrm{m})$, as compared with cells cultured under normal conditions. The results of western blotting demonstrated that BNIP3 expression was significantly upregulated in NP cells subjected to ND for $24 \mathrm{~h}$, which coincided with AIF translocation to the cell nucleus and alterations in cell viability and $\Delta \psi \mathrm{m}$. Furthermore, BNIP3 overexpression increased ND-induced NP cell death, whereas knockdown of BNIP3 or AIF abolished ND-induced NP cell death. In addition, BNIP3 overexpression increased AIF expression and BNIP3 knockdown decreased AIF expression in NP cells subjected to ND. In conclusion, ND induced NP cell death partially via activation of the BNIP3/AIF signalling pathway. These findings provide novel insights into the potential mechanisms underlying ND-induced death of NP cells during disc degeneration.
\end{abstract}

Correspondence to: Professor Jian Wang, Department of Orthopaedics, Xinqiao Hospital, The Third Military Medical University, 30 Center Street, Chongqing 400038, P.R. China E-mail: wangjian20161109@163.com

Abbreviations: AIF, apoptosis-inducing factor; ATP, adenosine triphosphate; BNIP3, B-cell lymphoma 2/adenovirus E1B $19 \mathrm{kDa}$ interacting protein; ND, nutrient deprivation; NP, nucleus pulposus; pNA, p-nitroaniline

Key words: nucleus pulposus cells, nutrient deprivation, cell death, B-cell lymphoma 2/adenovirus E1B $19 \mathrm{kDa}$-interacting protein 3, apoptosis-inducing factor

\section{Introduction}

Intervertebral disc degeneration disease is an important health problem worldwide, resulting in a large economic burden (1). During degeneration, the intervertebral discs exhibit extensive histomorphological alterations, including apoptosis of nucleus pulposus (NP) cells, lamellae disorganization of the annulus fibrosis, as well as thinning and calcification of the cartilaginous endplates $(2,3)$. Furthermore, the supply of nutrients, particularly oxygen and glucose, is significantly decreased and in some cases even non-existent. The increased rate of NP cell death during degeneration coincides with a decrease in nutrients supplied to the disc, indicating that a reduction in the supply of nutrients may be an important factor involved in NP cell death in human discs (4).

B-cell lymphoma 2 (Bcl-2)/adenovirus E1B 19-kDa-interacting protein 3 (BNIP3) is a member of a unique family of death-inducing mitochondrial proteins, which possesses a single Bcl-2 homology 3 (BH3) domain that acts as a pro-death factor (5). Under normal conditions, BNIP3 is expressed at low levels in various cell types, where it is primarily localized to the cytosol $(5,6)$. However, BNIP3 expression is increased under hypoxic conditions and is translocated from the cytosol to the mitochondria, where it causes mitochondrial dysfunction and subsequently induces cell death. Furthermore, apoptosis-inducing factor (AIF) is a key factor in caspase-independent cell death (7), which is anchored to the outer face of the inner mitochondrial membrane. In response to apoptotic stimuli, AIF is released into the cytosol and translocates to the nucleus, where it induces chromatin condensation and large-scale DNA fragmentation $(\sim 50 \mathrm{~kb})$ in a caspase 3 -independent manner $(8,9)$. Our previous study reported that BNIP3 expression was upregulated in NP cells in response to nutrient deprivation (ND) (10). However, the exact role of BNIP3 in ND-induced NP cell death, as well as the underlying mechanism, remains to be elucidated. Accordingly, the present study hypothesized that during disc degeneration, the nutrient supply induces alterations in the microenvironment of NP cells, which may increase BNIP3 expression and subsequently cause mitochondrial dysfunction, thereby resulting in the release and translocation of AIF to the nucleus, ultimately inducing apoptosis of NP cells. To confirm this hypothesis, the role of BNIP3 in ND-induced cell death and the potential involvement of AIF in this process were investigated. The present study is the first, 
to the best of our knowledge, to demonstrate that ND induced cell death of NP cells partially via activation of the BNIP3/AIF signalling pathway, thereby providing a novel insight into the potential mechanism underlying ND-induced NP cell death during disc degeneration.

\section{Materials and methods}

Isolation and culture of NP cells from caudal discs. All animal experiments conducted in the present study were approved by the Animal Care and Use Committee of the Third Military Medical University (Chongqing, China; approval no. SYXC-2015-00012) and were conducted in strict accordance with the recommendations outlined in the Guide for the Care and Use of Laboratory Animals adopted by the National Institutes of Health (11). Thirty male Sprague-Dawley rats (150-200 g; age, 8-10 weeks) were provided by the Laboratory Animal Centre of the Third Military Medical University (Chongqing, China). The animals were given free access to food and water, and were housed 3 mice/cage in a room maintained at a constant temperature $\left(23 \pm 1^{\circ} \mathrm{C}\right)$ and humidity $(60 \pm 1 \%)$ with a 12 -h light-dark cycle. All efforts were made to lessen suffering and to minimize the number of animals used. NP cells from Sprague-Dawley rats were isolated from caudal discs as previously described (10). The NP cells were removed from both halves of each disc using blunt forceps and pooled. Pooled NP cells were digested with $0.2 \%(\mathrm{w} / \mathrm{v})$ collagenase II for $4 \mathrm{~h}$ at $37^{\circ} \mathrm{C}$. The digested tissue and solution were then filtered through a Falcon $40-\mu \mathrm{m}$ filter to remove non-digested fragments and were washed three times by centrifugation ( $5 \mathrm{~min}$ at $1,118 \mathrm{x} \mathrm{g}$ ). Typically, cell cultures were maintained for 3 days either under normal control conditions [Dulbecco's modified Eagle's medium (DMEM) with $5 \mathrm{mM}$ glucose and $10 \%$ fetal bovine serum (FBS; all from Hyclone; GE Healthcare Life Sciences, Logan, UT, USA); $37^{\circ} \mathrm{C}, 21 \% \mathrm{O}_{2}$ ] or varying conditions for the ND groups (DMEM without FBS or glucose; $37^{\circ} \mathrm{C}, 21 \% \mathrm{O}_{2}$ ).

Cell viability and apoptosis assays. Cell viability was detected using the trypan blue exclusion method, as described previously (12). Apoptosis was detected using an Annexin Vfluorescein isothiocyanate (FITC)/propidium iodide (PI) Apoptosis kit (Thermo Fisher Scientific, Inc., Waltham, MA, USA) according to the manufacturer's instructions. The stained cells were analysed by flow cytometry; fluorescence emission was detected at 530 and $575 \mathrm{~nm}$, and excitation was detected at $488 \mathrm{~nm}$.

Separation of cytosolic, mitochondrial and nuclear fractions. The cytosolic, mitochondrial and nuclear fractions were isolated as described previously, with some modifications (13). Briefly, $2 \times 10^{7}$ cells were harvested by trypsinization following treatment and were washed once with PBS. Subsequently, the cells were digitonin-permeablilized for $5 \mathrm{~min}$ on ice at a density of $4 \times 10^{7} / \mathrm{ml}$ in cytosolic extraction buffer $(75 \mathrm{mM} \mathrm{NaCl}$, $1 \mathrm{mM} \mathrm{NaH} \mathrm{PO}_{4}, 8 \mathrm{mM} \mathrm{Na} \mathrm{HPO}_{4}, 250 \mathrm{mM}$ sucrose, $1 \mathrm{mM}$ phenylmethylsulfonyl fluoride, $5 \mu \mathrm{g} / \mathrm{ml}$ leupeptin, $5 \mu \mathrm{g} / \mathrm{ml}$ aprotinin and $0.05 \%$ digitonin). Cells were then transferred to a pre-cooled tissue homogenizer and homogenized 30 times using a tight pestle. The homogenized cells were centrifuged at $800 \mathrm{x}$ g for $5 \mathrm{~min}$ at $4^{\circ} \mathrm{C}$ and the supernatant was separated from the pellet comprising mitochondria and cellular debris. The supernatant containing cytoplasmic protein was further purified by centrifugation at $13,000 \mathrm{x} g$ at $4^{\circ} \mathrm{C}$ for $10 \mathrm{~min}$. The pellet, containing the mitochondrial fraction was resuspended in $50 \mu$ l homogenization buffer $(10 \mathrm{mM}$ Tris $\mathrm{HCl}, \mathrm{pH} 6.7$, $0.15 \mathrm{mM} \mathrm{MgCl}_{2}, 0.25 \mathrm{M}$ sucrose, $1 \mathrm{mM}$ PMSF and $1 \mathrm{mM}$ DTT). Mitochondrial fractions were stored at $-80^{\circ} \mathrm{C}$ until further use. For cytosolic and nuclear extraction, the K266-100 Nuclear/Cytosol Fractionation kit Biovision, Inc. (Milpitas, CA, USA) was used. Protein contents were determined using the Bradford assay with bovine serum albumin (Hyclone; GE Healthcare Life Sciences) as standard.

Cytofluorimetric analysis of mitochondrial transmembrane potential $(\Delta \psi m) . \Delta \psi \mathrm{m}$ was measured using the lipophilic cationic probe JC-1. After treatment, the cells were subjected to flow cytometry as described previously (14). The excitation wavelength was $490 / 525 \mathrm{~nm}$ and emission was monitored at 530/590 nm for JC-1 single probes and aggregates, respectively.

RNA interference. Small interfering RNA (siRNA) targeting BNIP3 (cat. no. sc-37452) and AIF (cat. no. sc-29194) along with control siRNA (cat. no. sc-44230) were purchased from Santa Cruz Biotechnology, Inc. (Dallas, TX, USA). NP cells $\left(2 \times 10^{5}\right)$ were transfected in serum-free DMEM with Lipofectamine 2000 transfection reagent ( $4 \mu \mathrm{g} /$ well; Thermo Fisher Scientific, Inc.) containing $100 \mathrm{nM}$ siRNA for 5-7 h at $37^{\circ} \mathrm{C}$ according to the manufacturer's protocol. Subsequently, the cells were cultured in DMEM for an additional $24 \mathrm{~h}$ at $37^{\circ} \mathrm{C}$. As previously indicated, cells were then subjected to $\mathrm{ND}$ for up to $72 \mathrm{~h}$, harvested, and underwent further analysis.

Plasmids and transfection. The pEGFP-N1-BNIP3 plasmid was designed by Invitrogen (Thermo Fisher Scientific, Inc.) as previously described (15). Cells $\left(2 \times 10^{5}\right)$ were cultured in antibiotic-free DMEM for $24 \mathrm{~h}$ at $37^{\circ} \mathrm{C}$ and were then transfected with BNIP3 and control plasmids (3 $\mu \mathrm{g} /$ well) using Opti-MEM ${ }^{\circledR}$ I reduced serum media (Hyclone; GE Healthcare Life Sciences) and Lipofectamine 2000 transfection reagent (4 $\mu \mathrm{g} /$ well; Thermo Fisher Scientific, Inc.) according to the manufacturer's protocol. A total of $24 \mathrm{~h}$ post-transfection, the cells were washed and subjected to ND for the indicated durations.

Western blot analysis. Protein samples (40-60 $\mu \mathrm{g}$; extracted as described in the Separation of cytosolic, mitochondrial and nuclearfractions subsection) were separated by $10 \%$ SDS-PAGE and transferred to polyvinylidene difluoride membranes, which were incubated at $4^{\circ} \mathrm{C}$ for $12 \mathrm{~h}$ with the following primary antibodies: Polyclonal anti-BNIP3 (cat. no. ab10433; dilution, 1:300; Abcam, Cambridge, MA, USA), monoclonal anti-AIF (cat. no. ab32516; 1:500; Abcam), anti-cytochrome $c$ oxidase IV (cat. no. ab14744; Cox IV; 1:10,000; Abcam), anti-histone H1 (cat. no. ab203337; 1:500; Abcam), and anti- $\beta$-actin (cat. no. A1978; 1:2,000; Sigma-Aldrich; Merck KGaA, Darmstadt, Germany). Subsequently, the membranes were incubated with a horseradish peroxidase-conjugated goat anti-rabbit immunoglobulin G secondary antibody (cat. no. ab150028; 1:10,000; Abcam) at $37^{\circ} \mathrm{C}$ for $2 \mathrm{~h}$. Immunoblotting was 
detected using an enhanced chemiluminescence kit (Beyotime Institute of Biotechnology, Haimen, China) and images were captured using a FluorChem 8900 imager (Beyotime Institute of Biotechnology). Western blots were semi-quantified using ImageJ v4.02 software (National Institutes of Health, Bethesda, MD, USA).

Caspase 3 activity analysis. The generation of p-nitroaniline (pNA) from the caspase tetrapeptide substrate Ac-DEVD-pNA (Beyotime Institute of Biotechnology) was used as an index of caspase 3 activity, as described previously (16). Optical density of free pNA was measured at an absorption wavelength of $405 \mathrm{~nm}$ using a microplate spectrophotometer (Dynex Technologies, Inc., Chantilly, VA, USA).

Statistical analysis. All values are presented as the mean \pm standard deviation. Differences between samples were assessed by one-way analysis of variance with a Bonferroni post hoc test for multiple comparisons using SPSS v22.0 software (IBM Corp., Armonk, NY, USA). P $<0.05$ was considered to indicate a statistically significant difference.

\section{Results}

ND induces NP cell death. The effects of ND on the viability of NP cells were determined using a trypan blue exclusion assay. The results detected a time-dependent decrease in viability of NP cells subjected to ND for up to $72 \mathrm{~h}$ (Fig. 1A). As determined using the Annexin V-FITC/PI apoptosis assay, the mode of cell death was predominantly apoptosis (Fig. 1B). Together, these data suggested that ND induced cell death, particularly apoptosis, of NP cells, which was consistent with the findings of our previous study (10).

ND-induced cell death is associated with increased BNIP3 expression and mitochondrial translocation in NP cells. To determine whether ND was able to alter BNIP3 expression, cells were subjected to ND for up to $72 \mathrm{~h}$ and protein expression was detected by western blot analysis. BNIP3 was expressed at a low level in the control cells, whereas ND significantly increased BNIP3 expression over the 72-h observation period (Fig. 2A and B); this exhibited a similar trend to the cell death response.

To examine how BNIP3 may induce NP cell death, the subcellular localization of ND-induced BNIP3 was detected. Differential centrifugation was used to prepare mitochondrial, cytosolic and nuclear fractions, which were probed with a BNIP3 antibody. As loading controls, the cytosolic fraction was probed with a $\beta$-actin antibody, the mitochondrial fraction with a Cox IV antibody and the nuclear fraction with a histone $\mathrm{H} 1$ antibody. As presented in Fig. 2C and D, ND increased the mitochondrial translocation of BNIP3. A small amount of BNIP3 was present in the cytosolic fraction, whereas none was observed in the nuclear fraction. In addition, the $\Delta \psi \mathrm{m}$ of cells cultured under normal and ND conditions was detected using JC-1. As shown in Fig. 2E, 93\% of the control cells exhibited double green-red fluorescence, whereas there was a time-dependent decrease in the percentage of ND-treated cells exhibiting double green-red fluorescence $(\sim 80 \%$ at $24 \mathrm{~h}, \sim 61 \%$ at $48 \mathrm{~h}$ and $\sim 15 \%$ at $72 \mathrm{~h}$ ), confirming a significant alteration
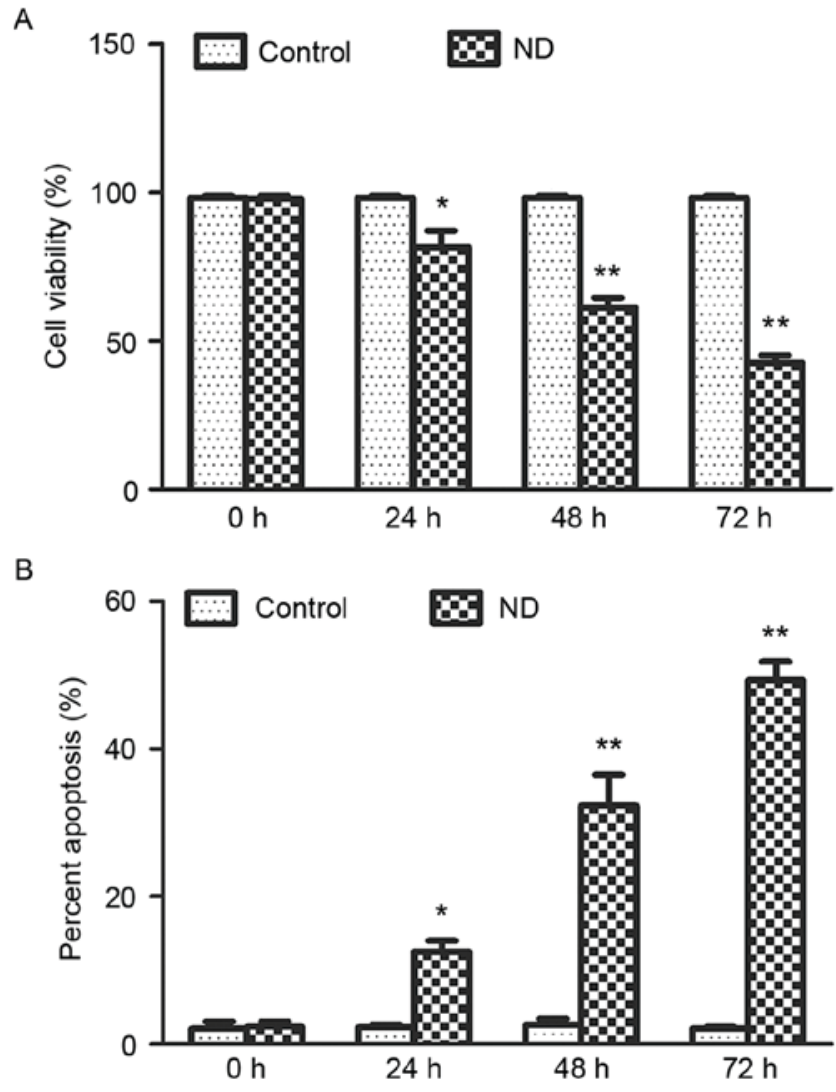

Figure 1. ND induces nucleus pulposus cell death. Cells were subjected to ND for up to $72 \mathrm{~h}$ and cell death was quantified. (A) Cell viability was determined using the trypan blue exclusion method. (B) Percentage of apoptotic cells was determined using the Annexin V-fluorescein isothiocyanate/propidium iodide assay. Data are presented as the mean \pm standard deviation of three experiments. ${ }^{*} \mathrm{P}<0.05,{ }^{* *} \mathrm{P}<0.01$ vs. the control group. ND, nutrient deprivation.

in $\Delta \psi \mathrm{m}$. These data suggested that ND may upregulate the expression of BNIP3 thereby inducing its translocation to mitochondria and causing mitochondrial dysfunction.

ND induces NP cell death in a BNIP3-dependent manner. In order to further determine the role of BNIP3 in ND-induced cell death, BNIP3 was overexpressed or silenced in NP cells using a transient transfection method. As shown in Fig. 3A and $\mathrm{B}, \mathrm{BNIP} 3$ protein expression was significantly upregulated in NP cells transfected with pEGFP-N1-BNIP3. Conversely, BNIP3 expression in BNIP3 siRNA-transfected cells was markedly lower than that of the control cells (Fig. 3C and D). Furthermore, ND-induced death of NP cells was significantly increased by BNIP3 overexpression (Fig. 3E and F). However, as expected, ND-induced NP cell death was markedly inhibited by BNIP3 knockdown (Fig. 3G and H). Together, these results suggested that ND stimulated NP cell death via BNIP3 regulation.

AIF serves a key role in BNIP3-activated cell death in ND-treated NP cells. The involvement of AIF in ND-induced NP cell death was investigated. As presented in Fig. 4A and B, the total amount of AIF did not vary with exposure time to ND. However, AIF expression in the mitochondrial fraction was decreased with exposure time to ND; expression began to markedly decrease after ND for $48 \mathrm{~h}$ and expression 
A

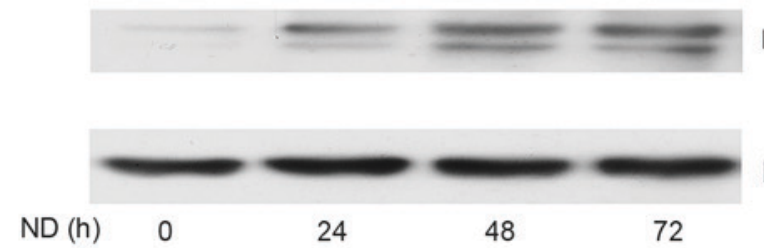

C

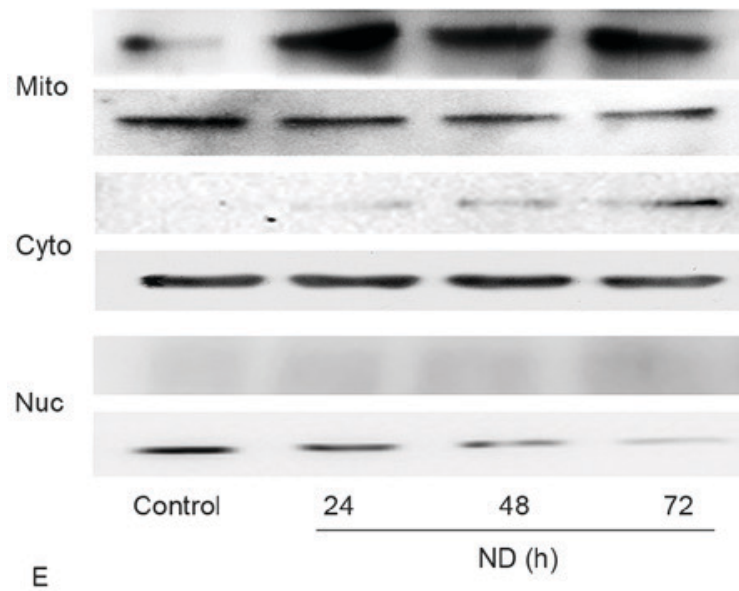

$\mathrm{E}$
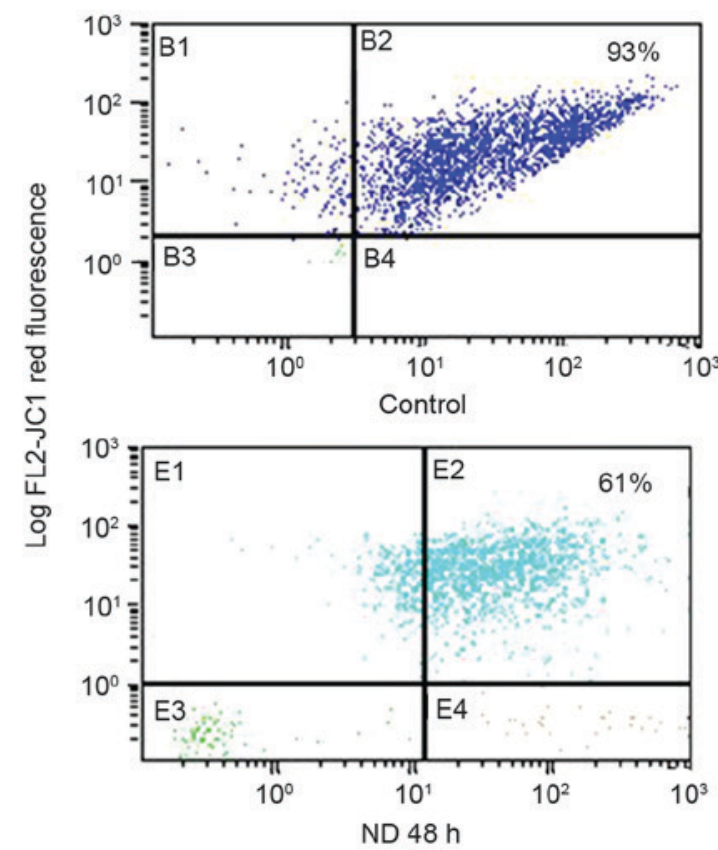

B

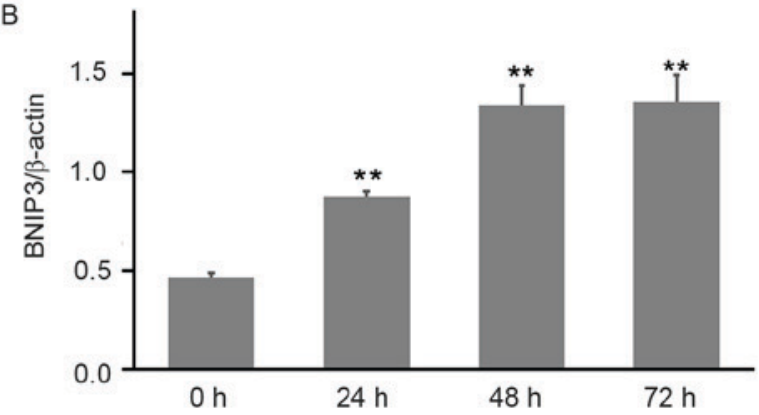

D
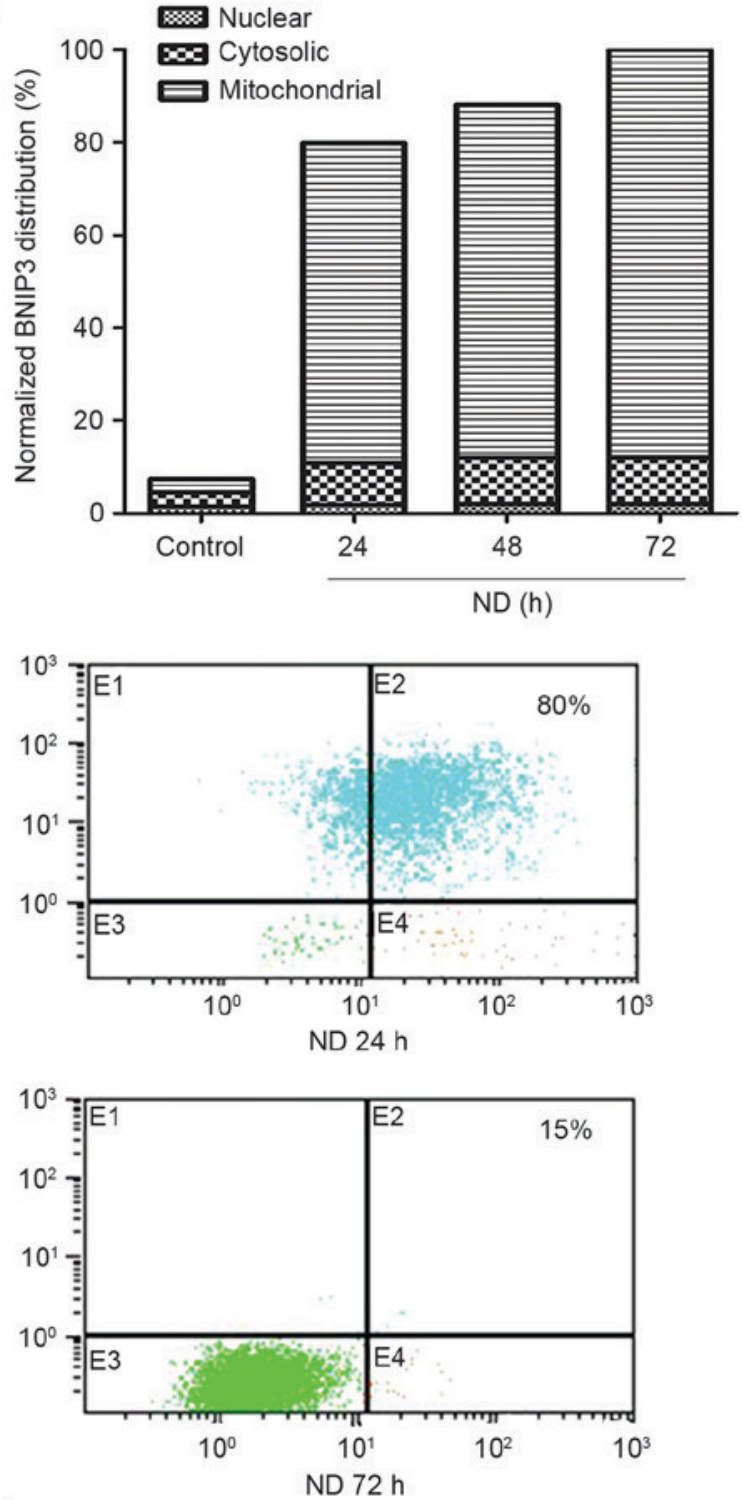

Log FL1-JC1 green fluorescence

Figure 2. ND-induced cell death is associated with increased BNIP3 expression and mitochondrial translocation in nucleus pulposus cells. Cells were subjected to ND for up to $72 \mathrm{~h}$. (A) Protein expression levels of BNIP3 were detected using western blot analysis. (B) BNIP3 protein expression was semi-quantified. (C) Protein extracts of cell fractions from control and treated cells were subjected to western blot analysis with an anti-BNIP3 antibody. (D) BNIP3 expression was normalized as the percentage of the total amount of BNIP3 at $72 \mathrm{~h}$. (E) Dot plot analyses of control and ND incubated with JC-1 and analysed by flow cytometry. Data are presented as the mean \pm standard deviation of three experiments. ${ }^{* *} \mathrm{P}<0.01$ vs. the vehicle-treated control group. BNIP3, B-cell lymphoma 2/adenovirus E1B 19 kDa-interacting protein; Cox IV, cytochrome $c$ oxidase IV; Cyto, cytoplasmic; Mito, mitochondrial; ND, nutrient deprivation; Nuc, nuclear.

had disappeared from the mitochondria after $72 \mathrm{~h}$ of ND. Furthermore, AIF expression was detected in the nuclear fraction after ND for $48 \mathrm{~h}$ and accumulated in a time-dependent manner, thus suggesting that AIF released from the mitochondria was translocated to the nucleus. In addition, BNIP3 overexpression increased the protein expression levels of AIF in NP cells exposed to ND, whereas BNIP3 knockdown inhibited ND-induced AIF expression (Fig. 4C and D). 
A

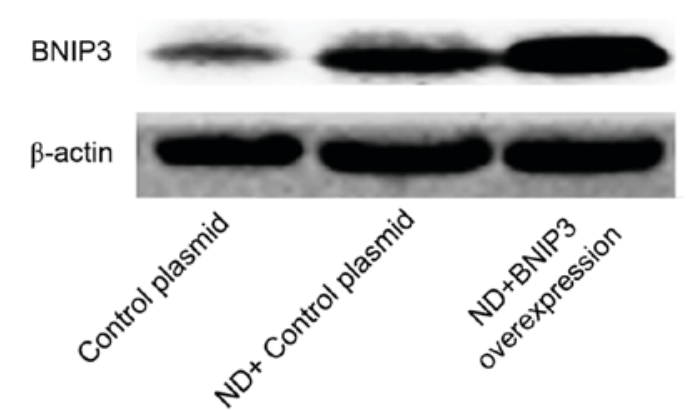

C

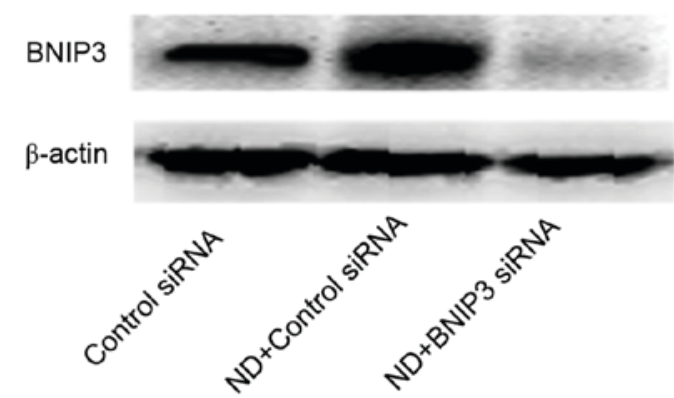

E

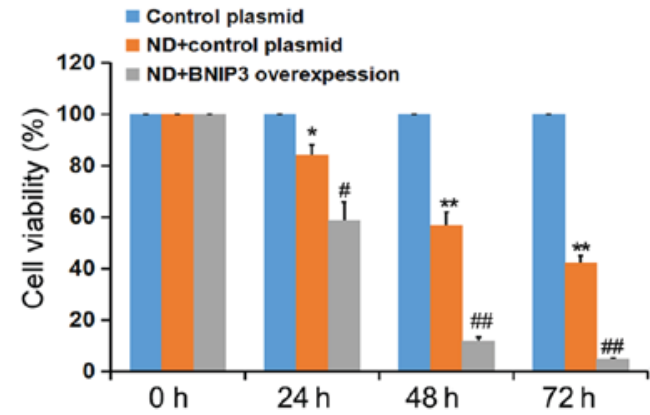

G

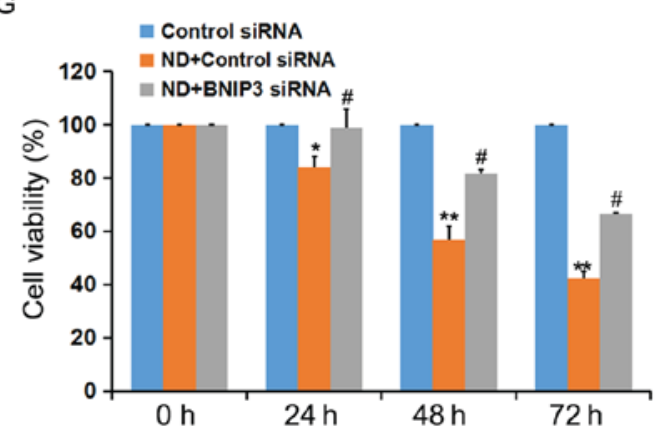

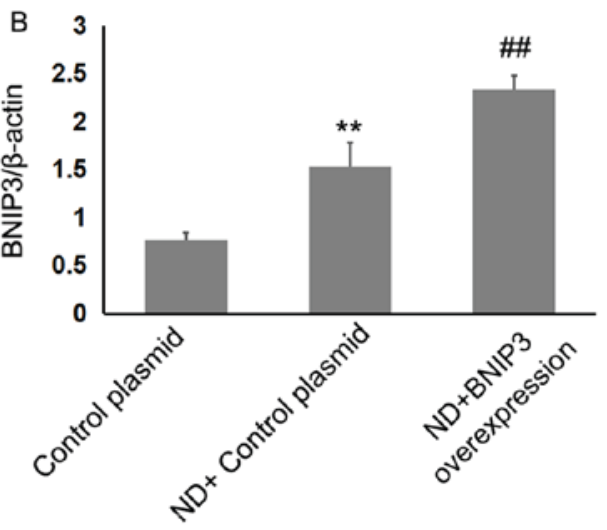

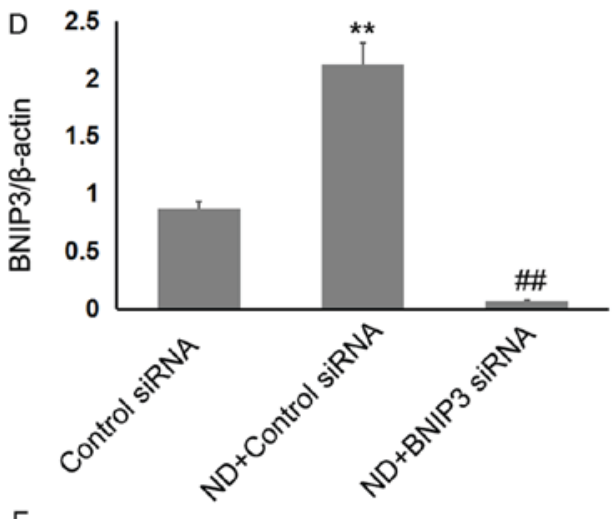

$\mathrm{F}$

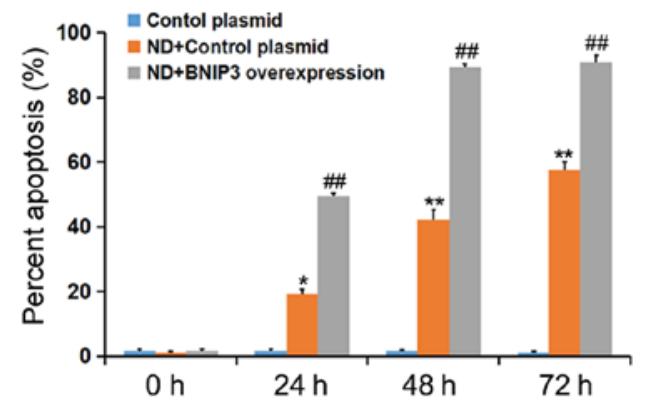

$\mathrm{H}$

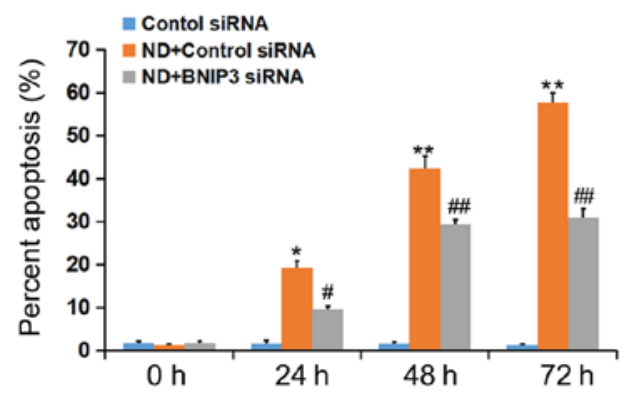

Figure 3. ND induces nucleus pulposus cell death in a BNIP3-dependent manner. NP cells were transfected with a plasmid expressing BNIP3 or with BNIP3 siRNA. After $24 \mathrm{~h}$, the cells were subjected to ND for a further $72 \mathrm{~h}$. (A and C) BNIP3 expression was detected by western blot analysis. (B and D) Bar graphs presenting the quantification of BNIP3. (E and F) Cell viability was determined by the trypan blue exclusion method. (G and H) Percentage of apoptotic cells was determined using the Annexin V-fluorescein isothiocyanate/propidium iodide assay. Data are presented as the mean \pm standard deviation of three experiments. ${ }^{~} \mathrm{P}<0.05,{ }^{* *} \mathrm{P}<0.01$ vs. the control group; ${ }^{"} \mathrm{P}<0.05,{ }^{\# \#} \mathrm{P}<0.01$ vs. the corresponding ND+control treated group (control siRNA or plasmid). BNIP3, B-cell lymphoma 2/adenovirus E1B $19 \mathrm{kDa}$-interacting protein; ND, nutrient deprivation; siRNA, small interfering RNA.

In order to further determine the involvement of AIF in BNIP3-induced death of NP cells exposed to ND, AIF siRNA was used to knockdown AIF expression. As shown in Fig. 4E and F, ND-induced BNIP3 expression was not affected by AIF
siRNA. However, ND-induced NP cell death was abolished following transfection with AIF siRNA (Fig. 4G and H). These results indicated that BNIP3-induced death of NP cells exposed to ND was dependent on AIF. 
A

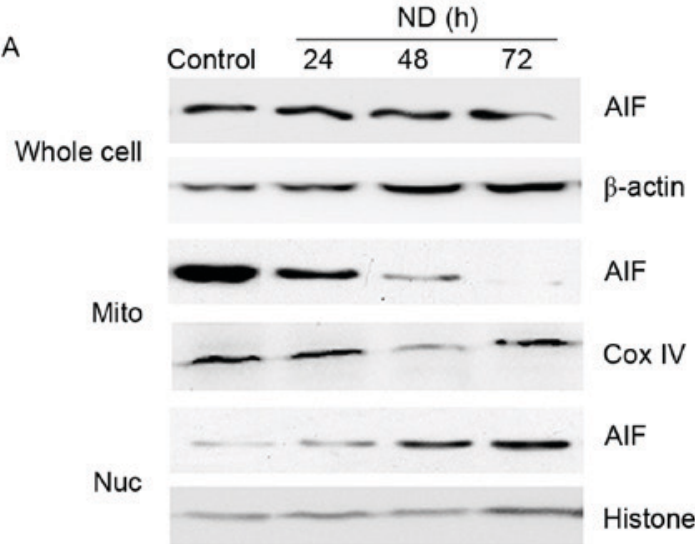

C

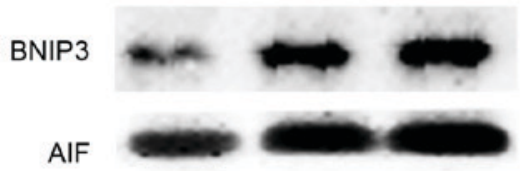

$\beta$-actin

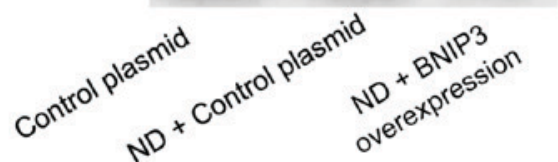

$\mathrm{E}$
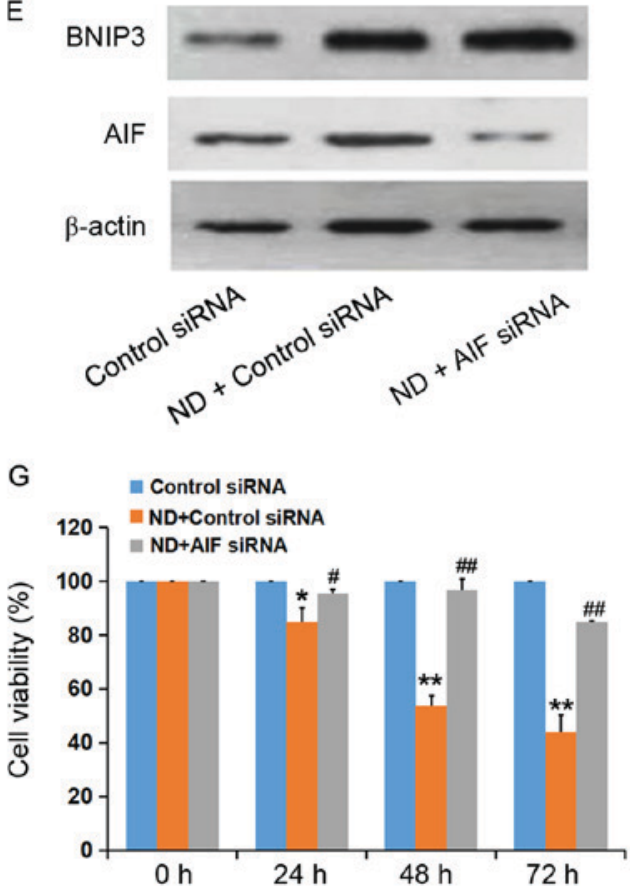

B

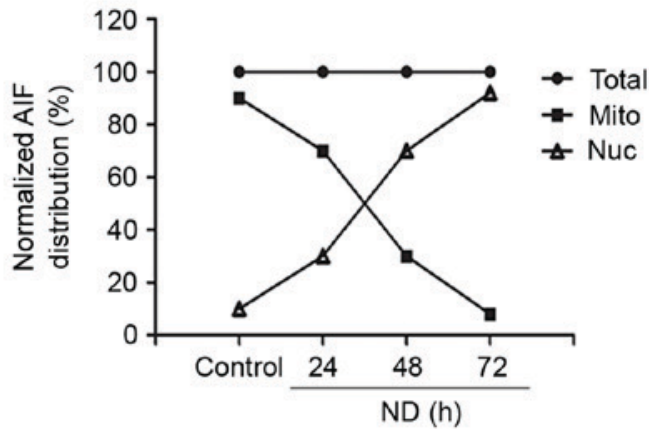

D
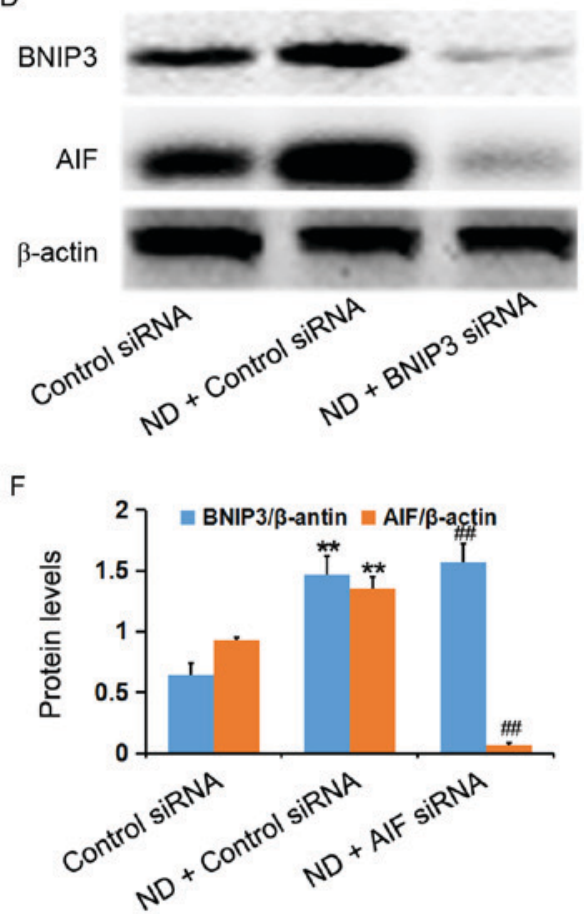

$\mathrm{H}$

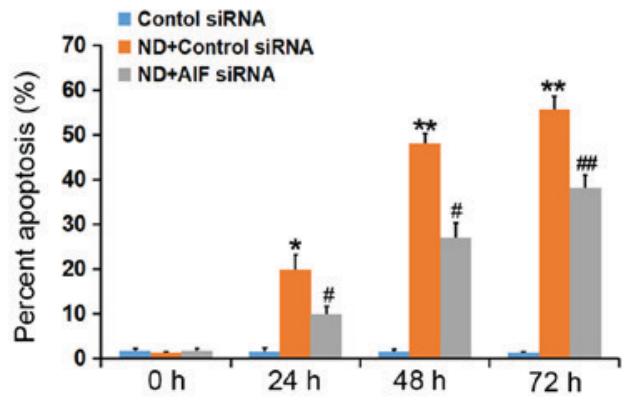

Figure 4. AIF serves a key role in BNIP3-induced death of nucleus pulposus cells exposed to ND. Cells were subjected to ND for up to 72 h. (A) Extracts of cell fractions from control and treated cells were subjected to western blot analysis with an anti-AIF antibody. (B) Levels of AIF distribution were normalized as the percentage of the total amount of AIF at each time. NP cells were transfected with either a plasmid expressing BNIP3 or with BNIP3 siRNA. Following $24 \mathrm{~h}$, the cells were subjected to ND for a further $72 \mathrm{~h}$. BNIP3 expression was detected in the (C) plasmid and (D) siRNA groups by western blot analysis. (E) AIF was knocked down by AIF siRNA. At $24 \mathrm{~h}$ post-transfection, the cells were subjected to ND for a further $72 \mathrm{~h}$. Cells were collected and lysed, and western blot analysis was performed. (F) Protein expression was semi-quantified for the siRNA groups. (G) Cell viability was determined using the trypan blue exclusion method. (H) Percentage of apoptotic cells was determined using the Annexin V-fluorescein isothiocyanate/propidium iodide assay. Data are presented as the mean \pm standard deviation of three experiments. ${ }^{*} \mathrm{P}<0.05,{ }^{* *} \mathrm{P}<0.01$ vs. the control group; ${ }^{\#} \mathrm{P}<0.05$, ${ }^{\# \#} \mathrm{P}<0.01 \mathrm{vs}$. the $\mathrm{ND}+\mathrm{control}$ siRNA-treated group. AIF, apoptosis-inducing factor; BNIP3, B-cell lymphoma 2/adenovirus E1B 19 kDa-interacting protein; Cox IV, cytochrome $c$ oxidase IV; Mito, mitochondrial; ND, nutrient deprivation; Nuc, nuclear; siRNA, small interfering RNA.

BNIP3-induced death of NP cells exposed to ND is independent of caspase 3. To determine whether the death of NP cells was caspase-dependent, caspase 3 activity was measured using a microplate spectrophotometer following ND. According to 
Table I. Results of caspase 3 activity assay.

\begin{tabular}{lc}
\hline Group & OD value \\
\hline Control & $0.036 \pm 0.005$ \\
ND $24 \mathrm{~h}$ & $0.028 \pm 0.002$ \\
ND $48 \mathrm{~h}$ & $0.027 \pm 0.004$ \\
ND $72 \mathrm{~h}$ & $0.032 \pm 0.008$ \\
\hline
\end{tabular}

OD value of caspase 3 activity was measured using a microplate spectrophotometer. Data are presented as the mean \pm standard deviation. ND, nutrient deprivation; OD, optical density.

the optical density values (Table I), during the 72-h culture period, caspase 3 activity was not significantly altered compared with in the control cells. These findings suggested that BNIP3-indcued NP cell death may be independent of caspase 3.

\section{Discussion}

Previous studies have reported that the initial characteristic of intervertebral disc degeneration appears to be associated with loss of NP cells, which indicates that NP cells serve an important role in the maintenance of disc health $(17,18)$. Understanding the mechanisms underlying NP cell death will help to further elucidate the pathomechanism of disc degeneration. It has previously been reported that a decrease in nutrient supply is the key factor leading to the death of NP cells during disc maturation and degeneration (19). However, the exact underlying mechanisms remain to be elucidated. Therefore, in the present study, a cell culture model under ND conditions was created to investigate the roles of BNIP3 (a nutrient-sensitive protein) and AIF (a mitochondrial pro-death protein) in the death pathway of NP cells.

Mitochondria serve an important role in cell death responses through the release of mitochondrial pro-death proteins, including AIF, endonuclease G and cytochrome $c$ (20). Members of the Bcl-2 family control the release of these proteins. Bcl-2 is an integral membrane protein that prevents apoptosis through inhibition of protein efflux, where BNIP3, $\mathrm{Bcl}-2$-associated $\mathrm{X}$ protein and $\mathrm{BH} 3$ interacting-domain death agonist are translocated from the cytoplasm to the outer mitochondrial membrane and trigger apoptosis upon release (21). As reported previously, the mitochondrial translocation of BNIP3 is involved in the ND-induced death of neurons (22). The results of the present study demonstrated that ND stimulated NP cell death via the regulation of BNIP3 expression and mitochondrial translocation. A previous study demonstrated that autophagy attenuates the catabolic effect of NP cells under inflammatory conditions, which is sustained by nuclear factor (NF)- $\kappa \mathrm{B}$ and inhibition of c-Jun N-terminal kinase (19). In addition, the stromal cell-derived factor-1/C-X-C motif chemokine receptor 4 axis promotes cell apoptosis in human degenerative NP cells via the NF- $\kappa \mathrm{B}$ pathway (20). To the best of our knowledge, the findings of the present study are the first to provide direct evidence of ND-induced NP cell death in a BNIP3-dependent manner, which is an important complement to previous studies.
Although caspase-dependent apoptosis is the main pathway of cell death, considerable evidence has suggested the importance of caspase-independent pathways (23-25). The mitochondrial apoptogenic factor AIF may induce apoptosis without cytochrome $c$-mediated caspase activation. In response to apoptotic stimuli, AIF is released into the cytosol and translocated to the nucleus where it induces chromatin condensation and large-scale DNA fragmentation (7). Notably, the results of the present study demonstrated that AIF was released from the mitochondria and translocated to the nucleus. Previous studies have indicated the need for a caspase 3 -dependent step in the release of $\operatorname{AIF}(26,27)$. However, the results of the present study reported that caspase 3 expression was not altered during ND-induced NP cell death. These results suggested that ND was capable of inducing the translocation of AIF into the nucleus during the progression of cell death in a caspase-independent manner.

It has previously been reported that BNIP3 is able to rapidly induce mitochondrial dysfunction by directly interacting with voltage-dependent anion channel/porin and adenine nucleotide translocase to stimulate opening of the mitochondrial permeability transition pore, thereby dissipating $\Delta \psi \mathrm{m}$ (28). The rapid loss of $\Delta \psi \mathrm{m}$ can lead to depletion of adenosine triphosphate (ATP), calcium ion imbalance, and eventual loss of plasma membrane integrity prior to subsequent execution of cell death (29). Another study demonstrated that AIF can also damage mitochondrial function, resulting in the loss of $\Delta \psi \mathrm{m}$ (30). It has also been indicated that the rapid and progressive depletion of intracellular ATP content triggers the disruption of $\Delta \psi \mathrm{m}$ in cells and induces caspase-independent apoptotic death (31). Therefore, the present study hypothesized that, in addition to the release of AIF, a BNIP3-mediated mitochondrial energetic failure may be associated with ND-induced NP cell death.

In conclusion, the present study is the first, to the best of our knowledge, to demonstrate that ND-induced NP cell death may partially occur via activation of the BNIP3/AIF signalling pathway. These results provide additional experimental evidence to elucidate the pathomechanism of intervertebral disc degeneration.

\section{Acknowledgements}

The present study was supported by the National Natural Science Foundation of China (grant nos. 81272028 and 81472131).

\section{References}

1. Dagenais S, Caro J and Haldeman S: A systematic review of low back pain cost of illness studies in the United States and internationally. Spine J 8: 8-20, 2008.

2. Ishii T, Tsuji H, Sano A, Katoh Y, Matsui H and Terahata N: Histochemical and ultrastructural observations on brown degeneration of human intervertebral disc. J Orthop Res 9: 78-90, 1991.

3. Thompson JP, Pearce RH, Schechter MT, Adams ME, Tsang IK and Bishop PB: Preliminary evaluation of a scheme for grading the gross morphology of the human intervertebral disc. Spine (Phila Pa 1976) 15: 411-415, 1990.

4. Ward E, Evans SE and Stern CD: The role of the somites and notochord in vertebral column development. Int J Exp Pathol 96: A11, 2015.

5. Vande Velde C, Cizeau J, Dubik D, Alimonti J, Brown T, Israels S, Hakem R and Greenberg AH: BNIP3 and genetic control of necrosis-like cell death through the mitochondrial permeability transition pore. Mol Cell Biol 20: 5454-5468, 2000. 
6. Lee H and Paik SG: Regulation of BNIP3 in normal and cancer cells. Mol Cells 21: 1-6, 2006.

7. Susin SA, Lorenzo HK, Zamzami N, Marzo I, Snow BE, Brothers GM, Mangion J, Jacotot E, Costantini P, Loeffler M, et al: Molecular characterization of mitochondrial apoptosis-inducing factor. Nature 397: 441-446, 1999.

8. Ye H, Cande C, Stephanou NC, Jiang S, Gurbuxani S, Larochette N, Daugas E, Garrido C, Kroemer G and Wu H: DNA binding is required for the apoptogenic action of apoptosis inducing factor. Nat Struct Biol 9: 680-684, 2002.

9. Cheung EC, Joza N, Steenaart NA, McClellan KA, Neuspiel M, McNamara S, MacLaurin JG, Rippstein P, Park DS, Shore GC, et al: Dissociating the dual roles of apoptosis-inducing factor in maintaining mitochondrial structure and apoptosis. EMBO J 25 : 4061-4073, 2006.

10. Liu J, Wang J and Zhou Y: Upregulation of BNIP3 and translocation to mitochondria in nutrition deprivation induced apoptosis in nucleus pulposus cells. Joint Bone Spine 79: 186-191, 2012.

11. Goodman JR: The Association For Assessment And Accreditation Of Laboratory Animal Care International fails to meaningfully address concerns regarding its accreditation program. J Appl Anim Welf Sci 18: 314-315, 2015.

12. Strober W: Trypan blue exclusion test of cell viability. Curr Protoc Immunol 111: A3.B.1-3, 2015.

13. Zhou X, Chen M, Zeng X, Yang J, Deng H, Yi L and Mi MT: Resveratrol regulates mitochondrial reactive oxygen species homeostasis through Sirt3 signaling pathway in human vascular endothelial cells. Cell Death Dis 5: e1576, 2014.

14. Santos JH, Hunakova L, Chen Y, Bortner C and Van Houten B: Cell sorting experiments link persistent mitochondrial DNA damage with loss of mitochondrial membrane potential and apoptotic cell death. J Biol Chem 278: 1728-1734, 2003.

15. Ahn BH, Kim HS, Song S, Lee IH, Liu J, Vassilopoulos A, Deng CX and Finkel T: A role for the mitochondrial deacetylase Sirt3 in regulating energy homeostasis. Proc Natl Acad Sci USA 105: 14447-14452, 2008

16. Carvour M, Song C, Kaul S, Anantharam V, Kanthasamy A and Kanthasamy A: Chronic low-dose oxidative stress induces caspase-3-dependent pkcdelta proteolytic activation and apoptosis in a cell culture model of dopaminergic neurodegeneration. Ann N Y Acad Sci 1139: 197-205, 2008.

17. Oegema TR Jr: The role of disc cell heterogeneity in determining disc biochemistry: A speculation. Biochem Soc Trans 30: 839-844, 2002.

18. Hunter CJ, Matyas JR and Duncan NA: The notochordal cell in the nucleus pulposus: A review in the context of tissue engineering. Tissue Eng 9: 667-677, 2003.

19. Pozdniakov AL and Khvylia SI: Role of the alimentary factor in the development of morphological changes in the blood vessels in an experiment. Vestn Akad Med Nauk SSSR: 65-70, 1986 (In Russian).
20. Zanna C, Ghelli A, Porcelli AM, Martinuzzi A, Carelli V and Rugolo M: Caspase-independent death of Leber's hereditary optic neuropathy cybrids is driven by energetic failure and mediated by AIF and Endonuclease G. Apoptosis 10: 997-1007, 2005.

21. Harris MH and Thompson CB: The role of the Bcl-2 family in the regulation of outer mitochondrial membrane permeability. Cell Death Differ 7: 1182-1191, 2000.

22. Zhang Z, Yang X, Zhang S, Ma X and Kong J: BNIP3 upregulation and EndoG translocation in delayed neuronal death in stroke and in hypoxia. Stroke 38: 1606-1613, 2007.

23. Yang TM, Qi SN, Zhao N, Yang YJ, Yuan HQ, Zhang B and Jin S: Induction of apoptosis through caspase-independent or caspase-9-dependent pathway in mouse and human osteosarcoma cells by a new nitroxyl spin-labeled derivative of podophyllotoxin. Apoptosis 18: 727-738, 2013.

24. Oishi S, Tsuji M, Hasegawa $\mathrm{H}$ and Oguchi K: High glucose induce apoptosis in a human lymphoma (U937) cells by a caspase-independent pathway. J Pharmacol Sci 112: 258p, 2010.

25. Madeo F, Carmona-Gutierrez D, Ring J, Büttner S, Eisenberg T and Kroemer G: Caspase-dependent and caspase-independent cell death pathways in yeast. Biochem Biophys Res Commun 382: 227-231, 2009.

26. Arnoult D, Gaume B, Karbowski M, Sharpe JC, Cecconi F and Youle RJ: Mitochondrial release of AIF and EndoG requires caspase activation downstream of Bax/Bak-mediated permeabilization. EMBO J 22: 4385-4399, 2003.

27. Arnoult D, Karbowski M and Youle RJ: Caspase inhibition prevents the mitochondrial release of apoptosis-inducing factor. Cell Death Differ 10: 845-849, 2003

28. Zhang HM, Cheung P, Yanagawa B, McManus BM and Yang DC BNips: A group of pro-apoptotic proteins in the Bcl-2 family. Apoptosis 8: 229-236, 2003.

29. Prabhakaran K, Li L, Mills EM, Borowitz JL and Isom GE: Up-regulation of uncoupling protein 2 by cyanide is linked with cytotoxicity in mesencephalic cells. J Pharmacol Exp Ther 314: 1338-1345, 2005.

30. Kang YH, Yi MJ, Kim MJ, Park MT, Bae S, Kang CM, Cho CK, Park IC, Park MJ, Rhee $\mathrm{CH}$, et al: Caspase-independent cell death by arsenic trioxide in human cervical cancer cells: Reactive oxygen species-mediated poly (ADP-ribose) polymerise- 1 activation signals apoptosis-inducing factor release from mitochondria. Cancer Res 64: 8960-8967, 2004.

31. Ballif BA and Blenis J: Molecular mechanisms mediating mammalian mitogen-activated protein kinase (MAPK) kinase (MEK)-MAPK cell survival signals. Cell Growth Differ 12: 397-408, 2001 